\title{
Evolution of ferroelastic domain walls during phase transitions in barium titanate nanoparticles
}

\author{
Jiecheng Diao $\odot,{ }^{1, *}$ Xiaowen Shi, ${ }^{3, \dagger}$ Tadesse A. Assefa $\odot,{ }^{2}$ Longlong Wu $\odot,{ }^{2}$ Ana F. Suzana $\odot,{ }^{2}$ Daniel S. Nunes, ${ }^{1}$ \\ Darren Batey, ${ }^{3}$ Silvia Cipiccia, ${ }^{3}$ Christoph Rau, ${ }^{3}$ Ross J. Harder $\odot,{ }^{4}$ Wonsuk Cha $\odot,{ }^{4}$ and Ian K. Robinson $\odot{ }^{1,2, *}$ \\ ${ }^{1}$ London Centre for Nanotechnology, University College London, London WC1E 6BT, United Kingdom \\ ${ }^{2}$ Condensed Matter Physics and Materials Science Department, Brookhaven National Lab, Upton, New York 11793, USA \\ ${ }^{3}$ Diamond Light Source, Oxfordshire, OX11 0QX, United Kingdom \\ ${ }^{4}$ Advanced Photon Source, Argonne National Lab, Lemont, Illinois 60439, USA
}

(Received 6 June 2020; revised 4 September 2020; accepted 11 September 2020; published 27 October 2020)

\begin{abstract}
In this work, ferroelastic domain walls inside $\mathrm{BaTiO}_{3}$ (BTO) tetragonal nanocrystals are distinguished by Bragg peak position and studied with Bragg coherent $\mathrm{x}$-ray diffraction imaging (BCDI). Convergence-related features of the BCDI method for strongly phased objects are reported. A ferroelastic domain wall inside a BTO crystal has been tracked and imaged across the tetragonal-cubic phase transition and proves to be reversible. The linear relationship of relative displacement between two twin domains with temperature is measured and shows a different slope for heating and cooling, while the tetragonality reproduces well over temperature changes in both directions. An edge dislocation is also observed and found to annihilate when heating the crystal close to the phase transition temperature.
\end{abstract}

DOI: 10.1103/PhysRevMaterials.4.106001

\section{INTRODUCTION}

Perovskite transition-metal oxides have been studied for decades because of both their broad applications and fundamental scientific questions. The displacement of $\mathrm{Ti}$ and $\mathrm{Ba}$ ions relative to the oxygen in unit cell leads to local polarization, which gives rise to exotic electrical properties such as elevated dielectric susceptibility, ferroelectricity, and piezoelectricity [1-4]. By analogy with well-studied magnetic systems, it is believed that it is not the local polarization in unit-cell level that directly links with these macroscopic electrical properties, but rather via the formation and rearrangement of polarized nanodomains. Therefore, the study of domain structures, preferably in three dimensions (3D), is important for understanding and improving these properties. $\mathrm{BaTiO}_{3}$ (BTO), for example, is frequently chosen as a lead-free functional material for both actuator and sensor applications [5,6]. It goes through a series of crystal lattice systems: cubic, tetragonal, orthorhombic, and rhombohedral upon cooling [7]. The corresponding transitions are first order with critical temperatures of 393,278 , and $183 \mathrm{~K}$, respectively, which can be adjusted by varying strain and sample size. The cubic-tetragonal phase transition temperature, for example, can be increased from 393 to $813 \mathrm{~K}$ with $1.7 \%$ compressive strain [8] and can decrease to room temperature when the particle size is reduced to $3 \mathrm{~nm}$ [9]. Recently, it was

\footnotetext{
*Corresponding author: jiecheng.diao.18@ucl.ac.uk

${ }^{\dagger}$ Present address: Department of Physics, New Mexico State University, Las Cruces, New Mexico 88003, USA and Department of Materials Science and Engineering, Rensselaer Polytechnic Institute, 110 8th Street, Troy, NY, 12180, USA.

‡Corresponding author: i.robinson@ucl.ac.uk
}

reported that the local structure remains locally rhombohedral throughout all phases [10,11].

The phase transition is also complex, demonstrating both order-disorder and displacive character [12,13]. From the high symmetry cubic phase to the lower symmetry tetragonal phase, the paraelectric ensemble breaks into ferroelectric domains of uniform electric polarization, driven by the minimization of the sum of electrostatic and elastic energy [1-4]. To accommodate local energy landscape and strain, different types of domains could be formed by rotation or translation of crystal regions or domains into different locations with well-defined domain-wall interfaces. For example, there are $71^{\circ}, 109^{\circ}$, and $180^{\circ}$ domain walls in rhombohedral BTO [14]. In tetragonal BTO, the flipping of one region of a crystal along a face diagonal leads to a ferroelectric and ferroelastic $90^{\circ}$ domain wall (twin boundary). While flipping along the long side of the tetragonal unit cell creates a ferroelectric-only $180^{\circ}$ domain wall instead, in which the $a$ domain and $c$ domain are formed head-to-tail with each other [15-17]. The domain wall is said to be continuous, which means it can only end in other domain walls or grain boundaries [18]. There are discontinuities of polarization in the perpendicular direction of domain walls, where the local displacements would be expected to accumulate. The formation of these domains depends strongly on boundary conditions, such as sample shape, while the size of domains in thin films has a square-root dependence on thickness, known as the Kittel scaling law [19].

Bragg coherent x-ray diffraction imaging (BCDI) is a synchrotron-based lensless imaging method, which is well adapted to studying nanocrystals in three dimensions (3D). It is capable of imaging the shape and mapping out inner strain without damaging the nanocrystal [20-22]. In the BCDI experiment, the $3 \mathrm{D}$ diffraction pattern is collected in reciprocal space and inverted to real space with advanced phase 
retrieval algorithms [20]. Usually, a single $h k l$ Bragg peak is selected in the reciprocal lattice with a total momentum transfer vector $\mathbf{Q}=h \mathbf{a}^{*}+k \mathbf{b}^{*}+l \mathbf{c}^{*}$. In a completely general way, this gives a 3D complex image of the crystal, capturing both its electron density function as the complex amplitude signal and a projection of the distortions as the corresponding phase signal. A simple linear relationship exists between the crystal displacement field $\mathbf{u}(\mathbf{r})$ and the observed image phase, $\phi(\mathbf{r})=\mathbf{Q} \bullet \mathbf{u}(\mathbf{r})$ [20]. The amplitude, representing the average electron density, contains information about crystallinity and its isosurface can be used to visualize the shape of the crystal. Any local displacement of the unit cells of the crystal parallel to $\mathbf{Q}$ will change the relative phase of the scattering from those unit cells relative to the rest of the crystal; when this occurs in regions large enough to be resolved the distortion can be visualized as a region or domain with a measurable phase appearing in the image. When the phase shift exceeds $2 \pi$, a proper phase unwrapping operation is needed to preserve the continuity of the displacement field.

The BCDI technique is sensitive to defects and has the unique ability to identify the dislocations and grain boundaries inside crystals by their characteristic strain (displacement) patterns [23-26]. The interpretation of the phase as a projection of the displacement field is analogous to the generalized phase approximation used to interpret transmission electron microscopy images [27]. There have already been several studies of BTO by BCDI motivated by the ability to see its important domain structures. An important example is the discovery of an interesting electric-field- driven vortex structure [28-30]. Here we take advantage of the unique properties of BCDI to investigate the domain structure and dislocations inside BTO nanocrystals upon crossing the cubic-tetragonal phase transition. In this work, we extend the BCDI method by considering the case of two nearby overlapping Bragg peaks, originating from different regions or domains of the same nanocrystal, to explore the structure and properties of the domain walls formed between them.

\section{EXPERIMENTAL METHODS}

Commercial BTO powders with a nominal size of $200 \mathrm{~nm}$ were diluted in a solution of tetraethyl orthosilicate and ethanol at a ratio of 1:75 in volume. This solution was then drop casted onto the silicon wafer and annealed in the furnace at around $700^{\circ} \mathrm{C}$ for $1 \mathrm{~h}$. This forms an amorphous $\mathrm{SiO}_{2}$ bonding matrix, which is a common procedure for fixing nanoparticles on to a substrate in BCDI experiments, in order to avoid its movement due to beam pressure [31].

Ex situ and in situ experiments reported in this work were performed at two beamlines specializing in BCDI. At Advanced Photon Source (APS), beamline 34-ID-C uses a Kirkpatrick-Baez mirror focusing system to match a $70 \times$ $30 \mathrm{um}^{2}$ spatially coherent beam at $55 \mathrm{~m}$ from the source to the size of the sample. This gives a good signal level from 200-nm BTO nanocrystals. At Diamond Light Source (DLS) beamline I-13-1, we used a 400-um aperture $220 \mathrm{~m}$ from the source to cut out a spatially coherent beam, which was focused by a Fresnel zone plate to the size of the sample. The signal level from this latter system was found to be at least ten times weaker. In both cases the sample was rotated in the coherent, monochromatic, focused beam and the diffraction pattern was collected on a Medipix-technology area detector. At APS, the detector distance was variable and for the measurement reported in this manuscript, we keep the detector at $D=0.45 \mathrm{~m}$. While at DLS, the detector was fixed at $D=2.8 \mathrm{~m}$.

When the beam is coherent over the dimensions of the sample, its diffraction pattern acquires interference fringes surrounding each Bragg peak. This diffraction pattern from the nanocrystal was measured in three dimensions at the 101 or 110 Bragg peaks by rotating the sample stage over a short range, typically \pm 0.1 to $\pm 0.5^{\circ}$. This rocking curve scan is equivalent to the $2 \mathrm{D}$ detector plane sweeping across the Ewald sphere and capturing the 3D volume of reciprocal space around the Bragg peak [20]. The amplitude of the diffracted $x$ ray is the square root of intensity, while the phase information is lost. This famous phase problem is solved by iterative algorithms, using the additional information obtained by oversampling the intensity distribution in the fringes of the coherent diffraction pattern surrounding the Bragg peak. In this work, a combination of error reduction and hybrid inputoutput algorithms are used to iteratively retrieve the phase until the error between the amplitude of the reconstruction and measured diffraction patterns decreased close to $0.1 \%$ [32].

\section{DOMAIN WALLS IN TETRAGONAL BTO NANOCRYSTALS}

At room temperature, the 200-nm BTO nanocrystals have tetragonal structure, in which case the $90^{\circ}$ and $180^{\circ}$ type domain walls (DWs) are favorable because they are mechanically compatible with each other without crystal misfit and are electrically neutral. $90^{\circ}$ type DWs separate ferroelastic and ferrolelectric domains, while $180^{\circ}$ type DWs separate pure polarization domains. Figure 1 shows an example of a crystal twin structure captured inside a single tetragonal BTO nanocrystal. Figures 1 (a) and 1(c) show the diffraction patterns of the 101 peak and the 110 peak from differently oriented tetragonal regions of the same crystal. The omega angle difference between the centers of mass (COM) of these two diffraction patterns is $0.6^{\circ}$. There are interference fringes crossing the entire reciprocal space region spanning between the two diffraction patterns centers seen on the area detector. Figure 1(b) shows the fringes on the detector frame at the angle in the middle of the two COMs. The observation of continuous fringes is a clear indication that the 110 and the 101 peaks come from the same coherence volume in real space, so that their diffraction patterns can interfere coherently. This identifies them as coming from substructures of the same nanoparticle. This is confirmed by gradually moving the sample piezo stages transverse to the beam direction, and seeing that the intensities of the two peaks increase and decrease simultaneously in a 100 -nm size $\mathrm{x}$-ray beam. This confirms the Bragg intensities are coming from the same nanoparticle, for which the intensity variation is attributed to crystal moving in and out of the x-ray beam.

The diffraction patterns of the two peaks were reconstructed separately. Figure 1(d) is the reconstructed image from the 101 diffraction pattern in Fig. 1(a), where the shape is plotted as an isosurface (single 3D contour level) of the amplitude and the surface is color-cued with the local value of 


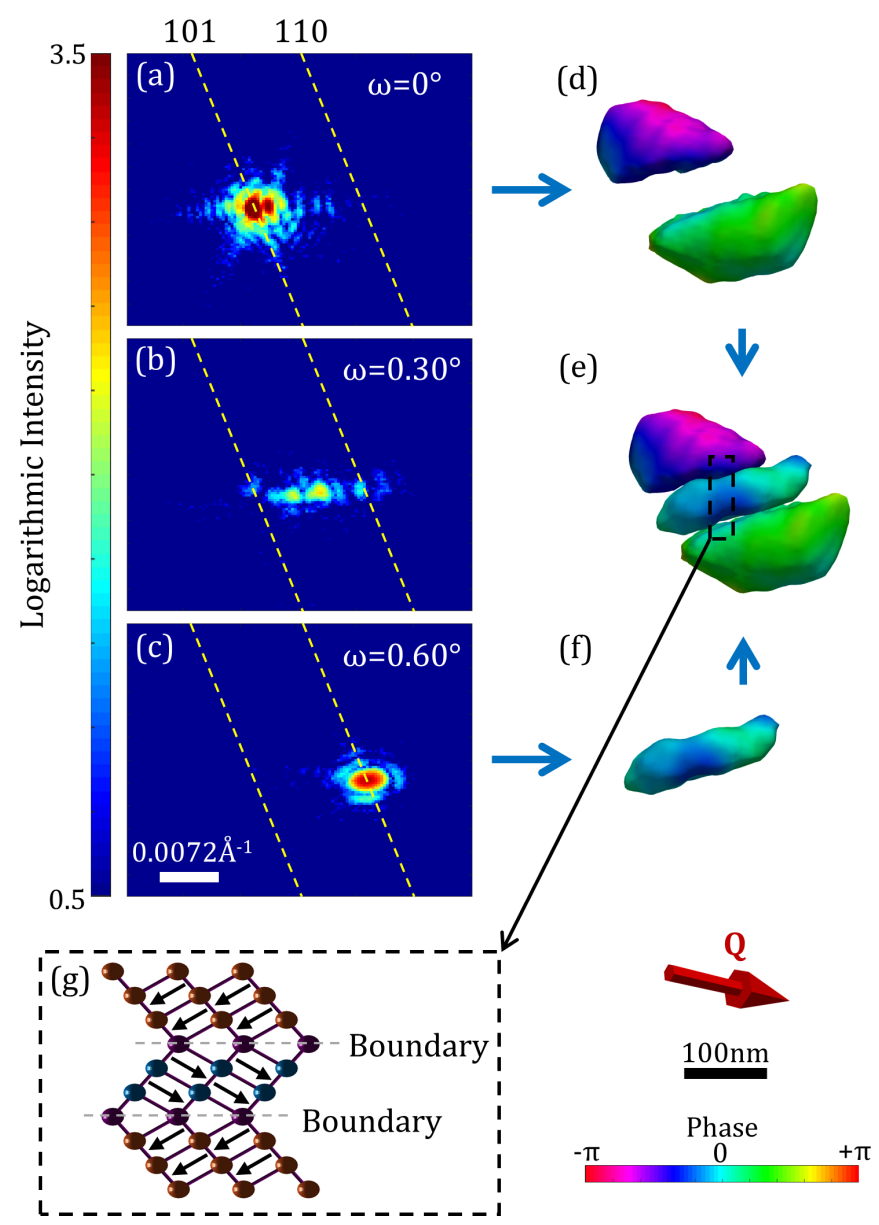

FIG. 1. Ferroelastic domain walls in $\mathrm{BaTiO}_{3}$ nanocrystal at room temperature. (a)-(c) Diffraction patterns of a $\mathrm{BaTiO}_{3}$ nanocrystal at the angles indicated. The location of the 101 and 110 powder rings on the area detector is plotted with yellow dashed lines as guides to the eye. Omega is the self-rotation angle of the sample stage. (d) and (f) Reconstructed images of 101 and 110 diffraction patterns in (a) and (c), respectively, shown as isosurfaces of amplitude to give the shape of crystal. The color on the shape of nanocrystal represents the complex phase, which can be reverted to displacement of crystal unit-cell origins. (e) A joint view of (d) and (f), which gives a good match in shape. $(\mathrm{g})$ A sketch of the two parallel ferroelastic domain walls inferred from these data at the position indicated by a black box in (e), which shows changes in the polarization direction upon crossing domain wall. The $Q$ direction is denoted, which is determined by the difference of the incident and diffracted $\mathrm{x}$-ray beam wave vectors. It denotes the Bragg reflection that was measured. The isosurface plots here and in the other figures were generated using the $3 \mathrm{D}$ visualization software PARAVIEW [33].

the phase. There are two separate domains with a 50-nm- wide gap in between. The two domains both have the same crystal orientation because they both contribute to the same 101 Bragg peak, but they have different phases [displacements, denoted by color in Fig. 1(d)]. This shows that the origins of their unit cells are shifted relative to each other. The average phases for the two 101 domains are $-1.259 \pm 0.004$ and 0.546 $\pm 0.003 \mathrm{rad}$, respectively. Because the phase can only be determined modulo $2 \pi$, this phase difference corresponds to a displacement of $2.835^{*} n+0.813 \AA$ between the two pieces along the $Q$ direction, where the $n$ is an integer. $2.835 \AA$ is the $\{101\} d$ spacing of BTO. The missing volume in between the two pieces implies there must be a third object, presumably an inserted piece of crystal with a different orientation, filling the gap. Figure 1(f), the reconstructed 3D image of the 110 pattern of Fig. 1(c), reveals a 50-nm plate-shaped crystal, which is the missing part. The two reconstructed images in Figs. 1(d) and 1(f) fit together well with each other, as shown in Fig. 1(e). We conclude that two $90^{\circ}$ domain walls exist in the same nanoparticle, which are parallel in this case. The polarization direction would change across a ferroelastic domain wall, in the way that is illustrated in Fig. $1(\mathrm{~g})$.

In analyzing the 3D diffraction data to obtain these BCDI reconstructions, it was found to be effective to arbitrarily cut clearly split diffraction peaks into two halves and to reconstruct the two peaks separately before reassembling them. The cropping of intermediary fringes between the two peaks did not seriously distort the resulting images, perhaps because the contribution of the fringes was at a low level relative to the peak centers. Further details of the influence of the cropping are presented in the Supplemental Material [34]. When the diffraction patterns of nanoparticles in Fig. 1 were reconstructed without splitting, shown in Fig. S3, they were found to be seriously misaligned with missing volumes in both cases. The combined double diffraction patterns, complete with the intermediary fringes, should still be the Fourier transform of the complete, assembled particle, so we would like to understand the reasons for this reconstruction failure. Similar misbehavior was reported for calculated diffraction patterns of a model nanocrystal containing an epitaxial interface between regions of different lattice constant [35]. We, therefore, undertook simulations of double diffraction patterns from known structures.

\section{SIMULATED BTO BICRYSTAL NANOPARTICLES}

Simulations were performed to test this behavior of the reconstruction algorithms, which are shown in Fig. 2. A model BTO nanoparticle, resembling that of Fig. 1, with three domains was built in a $512 \times 512 \times 512$ array. The two domains on the top and bottom have a hemispherical shape with a 32 pixels radius, while the middle domain has a cylinder shape that matches the two hemispheres with a height of 16 pixels. The object was Fourier transformed to generate its diffraction pattern, whose amplitude was then reconstructed using the usual algorithms [20]. The diffraction pattern and reconstructed images are presented in Figs. 2(a) and 2(e). Then the object was split into separate arrays for the hemispheres and the cylinder, separately Fourier transformed and then recombined as complex diffraction patterns to preserve the interference between the parts. When the two centers were the same, the result is shown in Fig. 2(a), while the split peak behavior was simulated offsetting the diffraction patterns before they were added together, using different gaps between the diffraction pattern from middle cylinder domain and the pattern from the two hemispheres, shown in Figs. 2(b)-2(d).

The 3D diffraction patterns in Figs. 2(a)-2(d) were recentered to their common COM before phase retrieval using the standard methods to give the images in Figs. 2(e)-2(h) [20]. Moving the two patterns apart from each other, making them 

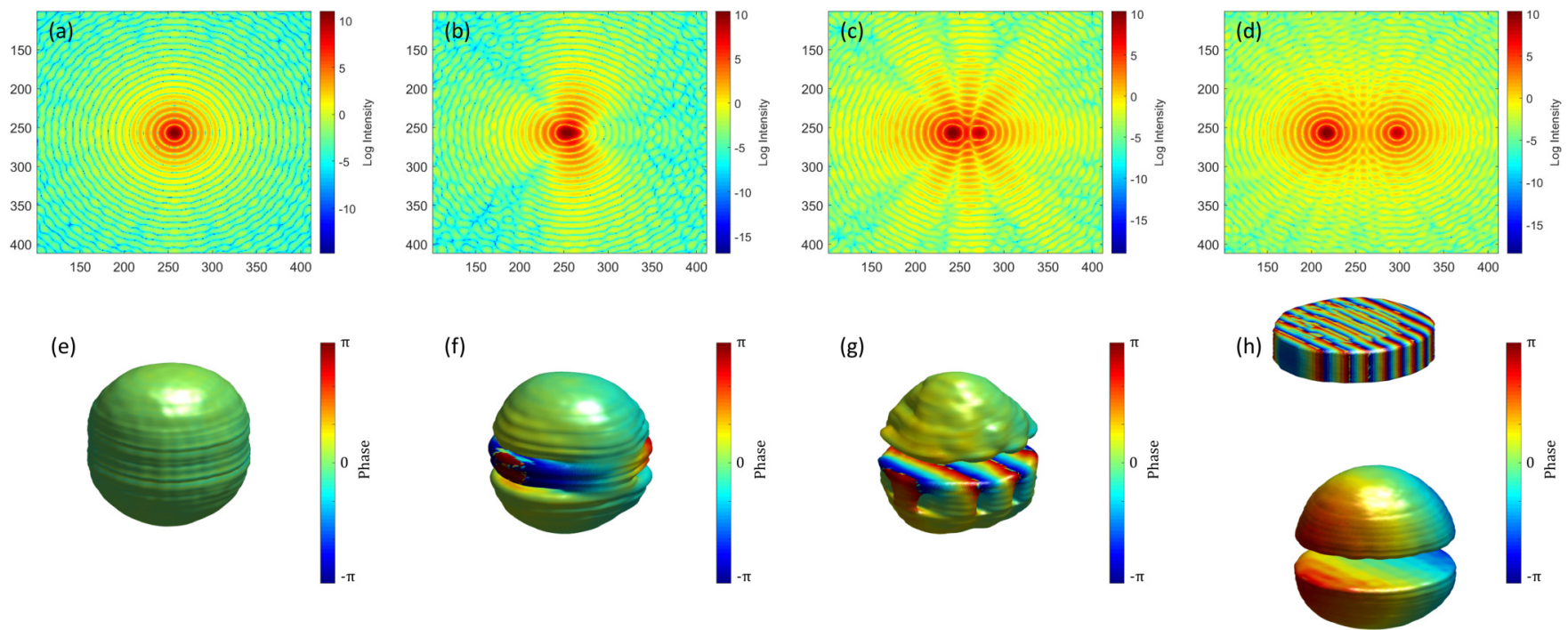

FIG. 2. Simulation of split peak diffraction pattern reconstructions. A nanoparticle with three domains was used to generate a 3D diffraction pattern of which the central slice is shown in a $512 \times 512$ pixel array. The two peaks corresponding to the hemispherical sides and cylindrical center were given an extra gap offsetting the two diffraction patterns by $0,5,15,40$ pixels from (a) to (d). Their reconstructed images are shown as isosurfaces colored by the image phase in (e) to (h), respectively.

misaligned from the array center, is equivalent to introducing phase ramps inside each of the particle segments in real space. From the definition of the discrete Fourier transform, it can be seen that each single pixel shift in reciprocal space corresponds to a $2 \pi$ phase ramp across the array in real space. When the gap between two patterns was 5 pixels in Figs. 2(b) and 2(f), the reconstruction was successful: the crystal still has the full shape, but there is an opposite phase gradient generated introduced in the different domains, positive for the cylinder and negative for the hemispheres. When the gap was increased to 15 pixels, in Figs. 2(c) and 2(g), the ramp becomes stronger, as expected, but the middle domain becomes misaligned and displaced into one side. There are missing crystal volumes inside the middle cylinder domain. Finally, when the gap reached 40 pixels, in Figs. 2(d) and 2(h), the middle domain becomes completely misaligned. Strong phase ramps and distorted density showed up in the middle cylinder domain and there is a phase gradient in two hemisphere domains.

This behavior of the reconstruction algorithms is presently unexplained, but the simulation results do reproduce the experimental behavior found in Fig. S3. We have nevertheless confirmed that the peak splitting is the cause of the apparent misalignment of reconstructed domains. It also justifies our strategy of reconstructing the split peak diffraction patterns separately and manually overlaying the images, which also avoids removing the phase ramps, which would have appeared otherwise. For the nanoparticle in Fig. 1, the splitting of two peaks, due to the twin boundary, gave separate reflections at 101 and 110 . The peak separation in reciprocal space is of the of order $0.02 \AA^{-1}$, which is considerably more than the 15 pixels needed to disrupt the correct reconstruction of the model ensemble nanoparticle in Fig. 2(c). This double peak reconstruction failure using the standard method is also reported in simulation works, where different algorithms are proposed to solve this problem [35].

\section{EVOLUTION OF FERROELASTIC DOMAIN WALLS UNDER PHASE TRANSITION}

A 200-nm BTO nanoparticle showing the twin-peak diffraction pattern was selected at $387.2 \mathrm{~K}$. Similar to the example in Fig. 1, it had its two peaks sitting on the 101 and 110 powder rings indicating the presence of an internal ferroelastic domain wall. Figure 3 shows the BCDI reconstruction, obtained directly from the double peak diffraction pattern, which clearly shows a $90^{\circ}$ domain wall inside. At this elevated temperature, the peaks were close enough to reconstruct together without requiring separating. The cross-section view in Fig. 3 shows the domain wall and two separated domains. The crystal planes of the two domains can be said to be parallel since they both have a constant phase inside and there is a sharp jump of the displacement field across the twin boundary. The phase ramps caused by splitting the peak is negligible compared with the large phase difference caused by domain walls, as indicated by the standard error of average displacement in the Supplemental Material [34]. This crystal also shows a small hole and spiral-shaped displacement distribution around the hole, which is an indication of a dislocation, whose details are discussed below. This nanoparticle was chosen to be tracked during heating across the tetragonal-to-cubic phase transition temperature, nominally at $393 \mathrm{~K}$.

Figure 4 shows a series of reconstructed images and a corresponding slice of this nanoparticle bicrystal going from tetragonal structure to cubic structure and back to tetragonal structure again by changing the temperature. When heating up the crystal before the phase transition, the shape of the crystal remains unchanged, but the relative displacement of the two domains (color) becomes small. After heating up to the cubic structure, the two domains are seen to merge into a single-color shape. Now in the cubic phase, the displacement differences have diminished, while new regions of both tensile and compressive strain have appeared on the surface of the im- 


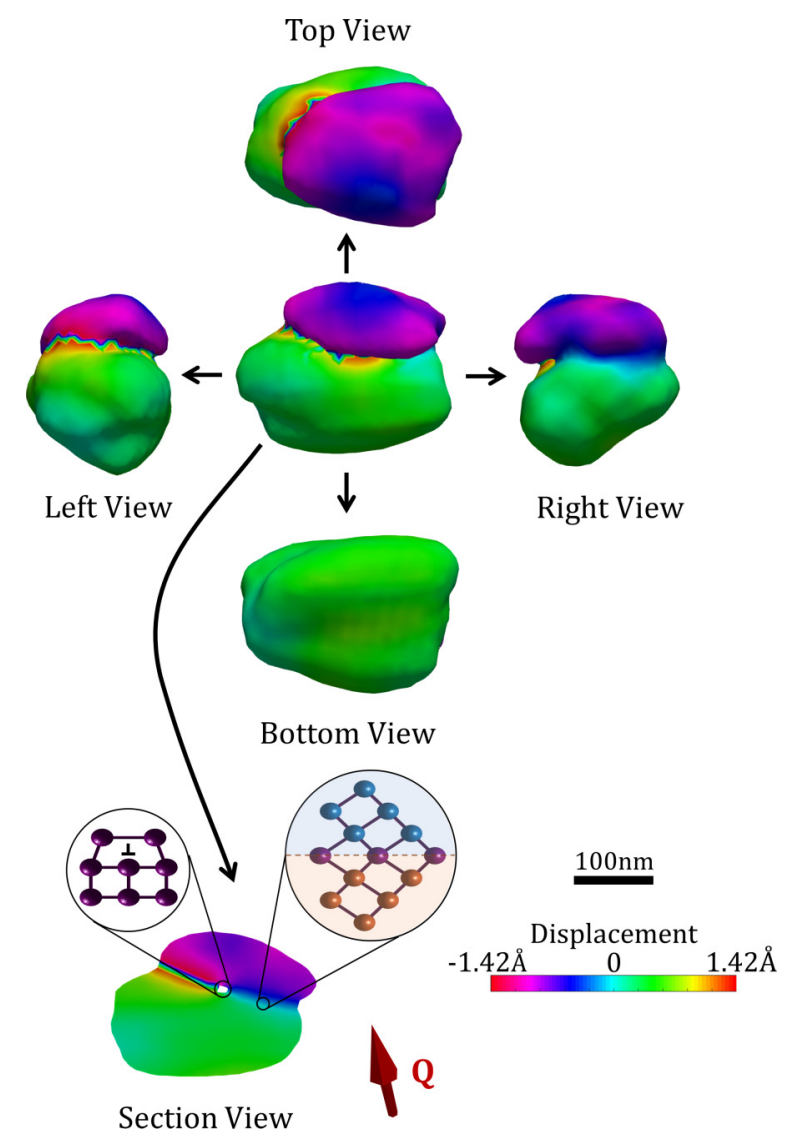

FIG. 3. BCDI isosurface image of a $\mathrm{BaTiO}_{3}$ nanoparticle containing two domains separated by a ferroelastic domain wall at 387.2 K. Five orthogonal views and a cross section are shown, as labeled, along with the $Q$ vector for the section view.

age. When the crystal is cooled down back into the tetragonal phase, the twin boundary comes back at the same position, so this transformation appears to be reversible, showing that the domain wall location is remembered by the nanoparticle.

The measured relative displacement of the two domains during heating up and cooling down are shown in Fig. 5(a) in a solid line. The displacement field decreases when in- creasing the temperature and vice versa. The standard error of each point is discussed in the Supplemental Material. Close to phase transition temperature, there appears to be a linear relationship of displacement difference with temperature on both heating and cooling. However, the slope during cooling is -0.21 , which is steeper than -0.48 while heating up. The goodness of fit is discussed in the Supplemental Material. The tetragonality of this crystal during heating and cooling, derived from the position of diffraction peak center, are shown in Fig. 5(a) in a dashed line. Comparing with both, the tetragonality reproduces well. However, there is a clear delay in the displacement field between cooling and heating. This difference in slope can be thought of as a form of hysteresis, commonly observed in phase transitions, coupled with the experimental limitation of waiting a sufficient time for the structure to equilibrate at each temperature.

To estimate the width of the domain wall, the displacement is plotted along a line passing vertically in Fig. 3 across the twin boundary in the region away from the dislocation, shown in Fig. 5(b). The width of the domain wall, where the sharp displacement slope could be seen, is below $30 \mathrm{~nm}$, which is the estimated spatial resolution of the image. The change of the phase across the step between the two crystals has a clear temperature dependence as the phase transition at $T=393 \mathrm{~K}$ is approached.

The displacement inside a domain is interpreted as the crystal distortion projected onto the $Q$ vector, or crystal plane displacement determined by the Bragg reflection. The average displacement between the ferroelastic domains is not caused by the accumulation of crystal distortion, but the structure of the ferroelastic domain wall instead. Because our spatial resolution is the same as the observed width of domain wall, the structure of the domain wall cannot be resolved and the displacement is smeared out at the domain wall position, as seen in Fig. 5(b). However, the relative displacement between the two domains is a good way to quantify the influence of a ferroelastic domain wall when the temperature changes. In this case, the relative displacement between the two domains is seen to increase linearly near the phase transition temperature. The reason for this change is not clear. There are possibilities that this change is coupled with the changing of

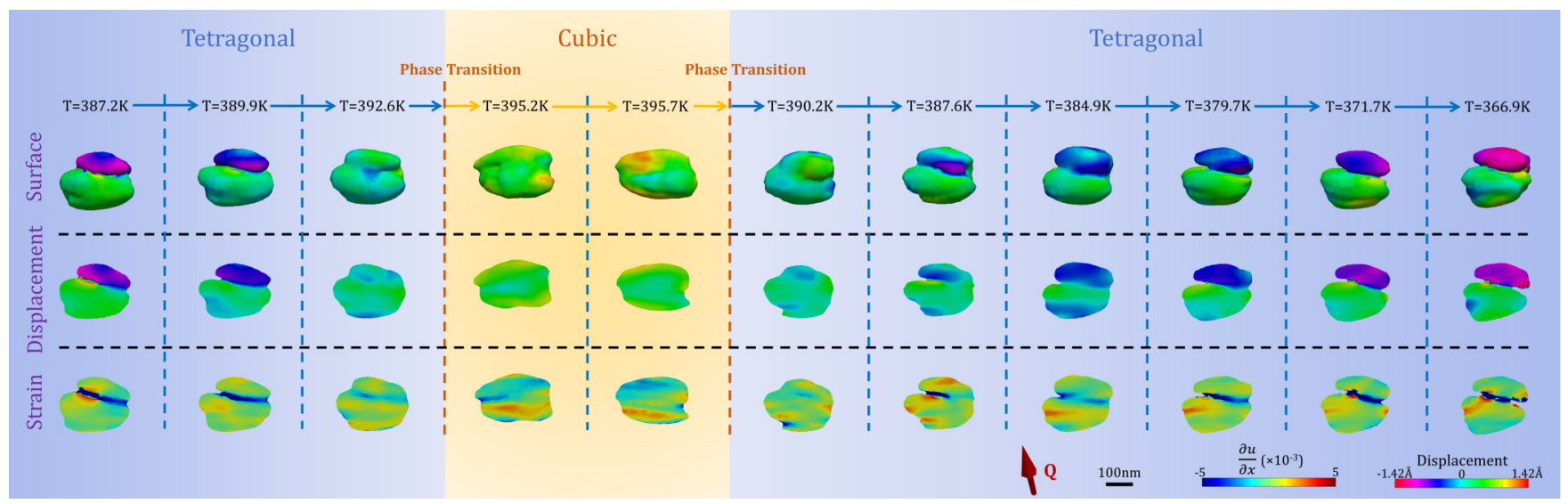

FIG. 4. Images of $\mathrm{BaTiO}_{3}$ nanoparticle upon crossing through its tetragonal-cubic phase transition. The top row is a series of contour views of the isosurface. The second row shows phase (displacement) cross-section maps taken in the middle of the nanocrystal, while the bottom row shows strain (a derivative of displacement) maps as a function of temperature. 

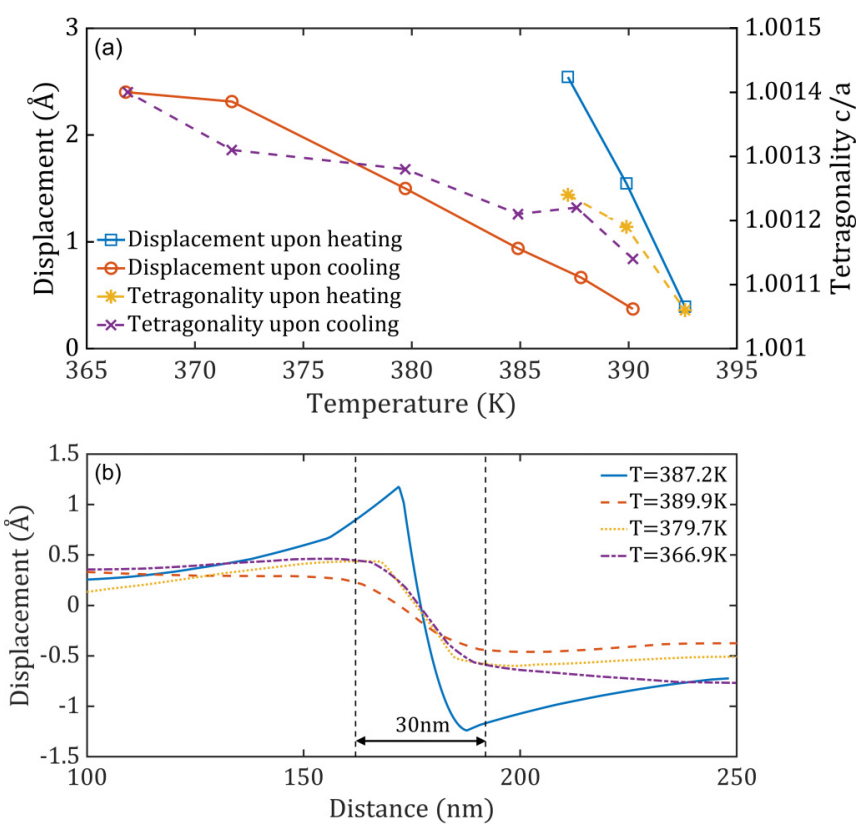

FIG. 5. (a) Average displacement difference between the two domains upon heating and cooling are shown by a solid line. Tetragonality of this crystal over heating and cooling are shown by a dashed line. (b) Line plot of displacement over distance across the twin boundary.

domain wall width, or the local distortion at the domain wall becomes sharper due to the change of tetragonality.

\section{DISLOCATION ANNIHILATION UPON HEATING}

Figures 6(a) and 6(b) show further details of the dislocation at the center of the ferroelastic domain wall inside the bicrystal. When the temperature is $387.2 \mathrm{~K}$, the dislocation is located at the center of crystal. The length of this dislocation is $178 \mathrm{~nm}$. Upon heating up to $389.9 \mathrm{~K}$, this dislocation is found to move to the left side and the length decreases to $125 \mathrm{~nm}$. Further heating up to $392.6 \mathrm{~K}$, although the crystal is still in the tetragonal phase, causes the dislocation to diminish again and then disappear. This dislocation does not come back during the cooling stage of the experiment.

To identify the type of dislocation, the displacement field surrounding the low electron density core is plotted as a function of rotation angle in Fig. 6(c). The experimental data show a roughly linear trend of crystal displacement over the angle superimposed with two clear modulations. This is the characteristic of an edge dislocation, for which is superimposed the simulated displacement field according to linear elastic theory, with details provided in the Appendix. The experimental data give a reasonable match with the simulated results.

\section{CONCLUSION}

We studied the structure and arrangement of domain walls in 200-nm BTO nanoparticles by BCDI both at ambient temperature and across the tetragonal-cubic phase transition temperature. Domains and domain walls are commonly found in these particles, giving rise to split coherent diffraction (a) $\mathrm{T}=387.2 \mathrm{~K}$

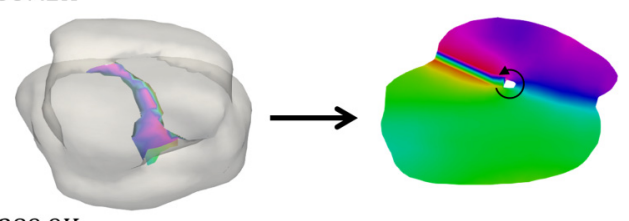

(b) $\mathrm{T}=389.9 \mathrm{~K}$
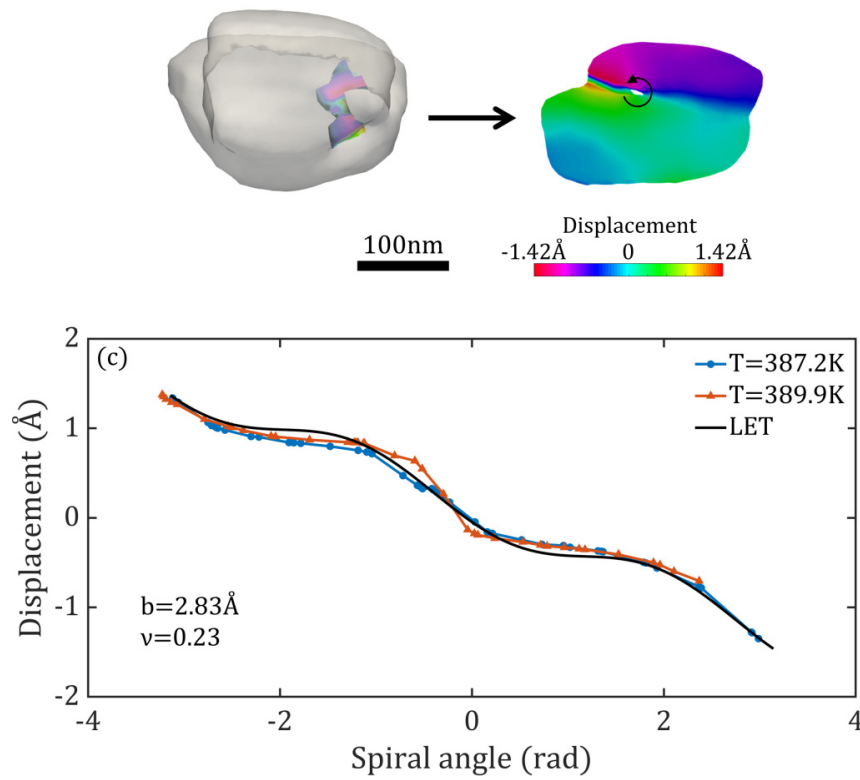

FIG. 6. Dislocation annihilation upon heating. (a) Reconstructed crystal image at $387.2 \mathrm{~K}$. (Left) A dislocation line through crystal is colored. (Right) A slice view across the dislocation line. (b) Same as (a), but temperature is 389.9 K. (c) Displacement field plotted vs rotation angle around the low-density core. Both experimental results and simulated curve from linear elastic theory are presented.

peaks. For successful BCDI reconstruction with large peak split, it was necessary to separate the peaks and manually reassemble the reconstructed 3D images afterwards. Ferroelastic domain walls were identified and characterized at room temperature. At temperatures close enough to the tetragonalcubic phase transition, the split peaks of a particle containing a ferroelastic domain wall were successfully reconstructed using both peaks together. This allowed us to determine the sub-Ångstrom relative displacement between two domains, tracking its disappearance on approaching the phase transition to cubic phase and reappearance when cooling back to tetragonal phase. We found a linear relationship between the relative displacement of the two $90^{\circ}$ domains over heating and cooling close to the tetragonal-cubic phase transition. The domain wall location was reproducible in this $200-\mathrm{nm}$ BTO nanoparticle. An edge dislocation line was found at the center of the twin boundary inside this crystal close to the transition temperature. This dislocation annihilated upon ramping up the temperature and did not return upon cooling.

\section{ACKNOWLEDGMENTS}

The work was supported by the UK EPSRC Grant No. EP/I022562/1 and the China Scholarship Council (CSC). 
Work at Brookhaven National Laboratory was supported by the U.S. Department of Energy (DOE), Office of Science, Office of Basic Energy Sciences, under Contract No. DESC0012704. Work at the Advanced Photon Source (APS) was supported by the U.S. DOE, Office of Science, Office of Basic Energy Sciences, under Contract No. DE-AC0206CH11357. The beamline 34-ID-C was built with funds from U.S. National Science Foundation Grant No. DMR-9724294. The authors acknowledge Diamond Light Source synchrotron facility for time on beamline I13-1.

\section{APPENDIX: IDENTIFICATION OF DISLOCATION IN FIGURE 6}

BCDI has the ability to locate the dislocation line inside a nanoparticle and identify the type of dislocation line by its characteristic strain field. The characteristic feature of any dislocation is a low-density core in the Bragg density map and a phase/displacement field around this core. A screw dislocation has a linear relation of displacement as a function of orientation angle given by [20]

$$
u_{\text {screw }}=\frac{b}{2 \pi} * \theta,
$$

where $u_{\text {screw }}$ is the displacement field around screw dislocation, $b$ is the Burgers vector, and $\theta$ is the spiral angle around screw dislocation.

An edge dislocation has linear relation plus two modulations given by the extra atomic planes [21]:

$$
u_{\text {edge }}=\frac{b}{2 \pi} *\left(\theta+\frac{\sin (2 \theta)-\cos (2 \theta)}{4-4 v}+\frac{(4 v-2) * \log ^{r}}{4-4 v}\right) \text {, }
$$

where $u_{\text {edge }}$ is the displacement field around the edge dislocation, $b$ is the Burgers vector, $\theta$ is the spiral angle around the edge dislocation, $r$ is the radius of the circle around the edge dislocation, and $v$ is Poisson's ratio of the material.

In this case, the simulated edge dislocation displacement field is plotted in Fig. 6(c). The radial distance $r$ is $30 \mathrm{~nm}$ and Poisson's ration is 0.23 . The value of the Burgers vector is $2.83 \AA$, which is the $d$ spacing of the corresponding lattice plane.
[1] P. Zubko, S. Gariglio, M. Gabay, P. Ghosez, and J.-M. Triscone, Annu. Rev. Condens. Matter Phys. 2, 141. (2011).

[2] D. G. Schlom, L. Q. Chen, C. B. Eom, K. M. Rabe, S. K. Streiffer, and J.-M. Triscone, Annu. Rev. Mater. Res. 37, 589 (2007).

[3] A. R. Damodaran, J. C. Agar, S. Pandya, Z. Chen, L. Dedon, R. $\mathrm{Xu}, \mathrm{B}$. Apgar, S. Saremi, and L. W. Martin, J. Condens. Matter Phys. 28, 263001 (2016).

[4] C. H. Ahn, K. M. Rabe, and J.-M. Triscone, Science 303, 488 (2004).

[5] P. Keller, H. Ferkel, K. Zweiacker, J. Naser, J.-U. Meyer, and W. Riehemann, Sens. Actuators B Chem. 57, 39 (1999).

[6] J. Gao, D. Xue, W. Liu, C. Zhou, and X. Ren, Actuators 6, 24 (2017).

[7] A. von Hippel, Rev. Mod. Phys. 22, 221 (1950).

[8] K. J. Choi, M. Biegalski, Y. L. Li, A. Sharan, J. Schubert, R. Uecker, P. Reiche, Y. B. Chen, X. Q. Pan, V. Gopalan, L.-Q. Chen, D. G. Schlom, and C. B. Eom, Science 306, 1005 (2004).

[9] J. E. Spanier, A. M. Kolpak, J. J. Urban, I. Grinberg, L. Ouyang, W. S. Yun, A. M. Rappe, and H. Park, Nano. Lett. 6, 735 (2006).

[10] J. Hlinka, T. Ostapchuk, D. Nuzhnyy, J. Petzelt, P. Kuzel, C. Kadlec, P. Vanek, I. Ponomareva, and L. Bellaiche, Phys. Rev. Lett. 101, 167402 (2008).

[11] Y. Isohama, N. Nakajima, H. Maruyama, Y. Tezuka, and T. J. Iwazumi, Electron Spectrosc. 184, 207 (2011).

[12] E. A. Stern, Phys. Rev. Lett. 93, 037601 (2004).

[13] M. B. Smith, K. Page, T. Siegrist, P. L. Redmond, E. C. Walter, R. Seshadri, L. E. Brus, and M. L. Steigerwald, J. Am. Chem. Soc. 130, 6955 (2008).

[14] M. Taherinejad and D. Vanderbilt, Phys. Rev. Lett. 86, 155138 (2012).

[15] A. S. Everhardt, S. Matzen, N. Domingo, G. Catalan, and B. Noheda, Adv. Electron. Mater. 2, 1500214 (2016).
[16] A. Schilling, T. B. Adams, R. M. Bowman, J. M. Gregg, G. Catalan, and J. F. Scott, Phys. Rev. B 74, 024115 (2006).

[17] L. J. McGilly, A. Schilling, and J. M. Gregg, Nano Lett. 10, 4200 (2010).

[18] G. Catalan, Physics of Ferroic and Multiferroic Domain Walls, in Mesoscopic Phenomena in Multifunctional Materials, Springer Series in Materials Science (Springer, Berlin, Heidelberg, 2014), Vol. 198.

[19] G. Catalan, J. Seidel, R. Ramesh, and J. F. Scott, Rev. Mod. Phys. 84, 119 (2012).

[20] I. Robinson and R. Harder, Nat. Mater. 8, 291 (2009).

[21] G. Xiong, O. Moutanabbir, M. Reiche, R. Harder, and I. Robinson, Adv. Mater. 26, 7747 (2014).

[22] T. A. Assefa, Y. Cao, J. Diao, R. J. Harder, W. Cha, K. Kisslinger, G. D. Gu, J. M. Tranquada, M. P. M. Dean, and I. K. Robinson, Phys. Rev. B 101, 054104 (2020).

[23] J. N. Clark, J. Ihli, A. S. Schenk, Y.-Y. Kim, A. N. Kulak, J. M. Campbell, G. Nisbet, F.C. Meldrum, and I. K. Robinson, Nat. Mater. 14, 780 (2015).

[24] A. Ulvestad, A. Singer, J. N. Clark, H. M. Cho, J. W. Kim, R. Harder, J. Maser, Y. S. Meng, and O. G. Shpyrko, Science 348, 1344 (2015).

[25] S. Labat, M. I. Richard, M. Dupraz, M. Gailhanou, G. Beutier, M. Verdier, F. Mastropietro, T. W. Cornelius, T. U. Schulli, J. Eymery, and O. Thomas, ACS Nano 9, 9210 (2015).

[26] X. Huang, W. Yang, R. Harder, Y. Sun, M. Lu, Y. S. Chu, I. Robinson, and H. K. Mao, Nano Lett. 15, 7644 (2015).

[27] M. Hytch, F. Houdellier, F. Hue, and E. Snoeck, Nature (London) 453, 1086 (2008).

[28] D. Karpov, Z. Liu, T. dos Santos Rolo, R. Harder, P. V. Balachandran, D. Xue, T. Lookman, and E. Fohtung, Nat. Commun. 8, 280 (2017).

[29] K. Ohwada, K. Sugawara, T. Abe, T. Ueno, A. Machida, T. Watanuki, S. Ueno, I. Fujii, S. Wada, and Y. Kuroiwa, Jpn. J. Appl. Phys. 58, SLLA05 (2019). 
[30] R. Harder and I. Robinson, JOM 65, 1202 (2013).

[31] M. Monteforte, A. K. Estandarte, B. Chen, R. Harder, M. H. Huang, and I. Robinson, J. Synchrotron Rad. 23, 953 (2016).

[32] J. R. Fienup, Appl. Opt. 21, 2758 (1982).

[33] J. Ahrens, B. Geveci, and C. Law, ParaView: An end-user tool for large data visualization, in Visualization Handbook (Elsevier, Amsterdam, 2005).
[34] See Supplemental Material at http://link.aps.org/ supplemental/10.1103/PhysRevMaterials.4.106001 for the influence of fringe cropping on the reconstructed images and details of the calculation of the displacement differences between two domains.

[35] Z. Wang, O. Gorobtsov, and A. Singer, New J. Phys. 22, 013021 (2020). 